\title{
Silicon Germanium Platform Enabling Mid-Infrared to Near- Infrared Conversion for Telecom and Sensing Applications
}

\author{
Bogris Adonis ${ }^{(1),(2)}$, Alexandros Kapsalis ${ }^{(2)}$, Kamal Hammani( ${ }^{(3)}$, Mohamed A. Ettabib ${ }^{(4)}$, Mickael \\ Brun $^{(4)}$, Pierre Labeye ${ }^{(4)}$, Sergio Nicoletti ${ }^{(4)}$ and Periklis Petropoulos ${ }^{(4)}$, Dimitris Syvridis ${ }^{(2)}$ \\ (1) Department of Informatics, Technological Educational Institute of Athens, Aghiou Spiridonos, 12210 \\ Egaleo, Athens, Greece, abogris@teiath.gr \\ (2) Department of Informatics and Telecommunications, National and Kapodistrian University of \\ Athens, Panepistimiopolis, llissia, 15784, Athens, Greece \\ (3) Laboratoire Interdisciplinaire Carnot de Bourgogne (ICB), UMR 6303 CNRS-Université de Bour- \\ gogne, 9 av. A. Savary, BP 47 870, F-21078 Dijon Cedex, France \\ (4) Optoelectronics Research Centre, University of Southampton, Highfield, Southampton, SO17 1BJ, \\ United Kingdom \\ (5) CEA-Leti MINATEC Campus, 17 rue des Martyrs 38054 Grenoble Cedex 9, France
}

\begin{abstract}
This paper presents the potential of silicon germanium waveguides in the nonlinear conversion of light from mid-infdrared wavelengths to the telecom band utilizing four-wave mixing. Design aspects and first characterization results of fabricated devices are presented.
\end{abstract}

\section{Introduction}

The mid-infrared (mid-IR) spectral region has attracted a significant amount of interest in recent years. It contains the absorption "fingerprints" of most molecules of interest, thus suggesting several applications both in spectroscopy and chemical and biomolecular sensing. The recent development of mid-IR sources, most notably quantum cascade lasers (QCLs) [1], has further stimulated the interest of the research community in this area. However, the lack of small size, room temperature detectors exhibiting suitably high sensitivity at this wavelength region still remains an issue.

To overcome this difficulty, wavelength conversion of the mid-IR signals into the near-infrared (near-IR) is a promising alternative. This conversion can be done either through sumfrequency generation in a second-order nonlinear medium, such as periodically poled lithium niobate [2], or through four-wave mixing (FWM) using a third-order nonlinear medium, such as silicon [3]. Silicon is an excellent candidate for such applications, thanks to its transparency up to $8 \mu \mathrm{m}$, the reduced two-photon and free-carrier absorptions beyond $2 \mu \mathrm{m}$ and its potential for monolithic integrated solutions. These features however, are not restricted to pure silicon. For instance, silicon germanium (SiGe) alloys have been identified as promising candidates for nonlinear applications in the midwave and longwave infrared thanks to their enhanced nonlinearity compared to pure Si [4]. Very recently [5], we have demonstrated that $\mathrm{Si}_{1-x} \mathrm{Ge}_{\mathrm{x}}$ waveguides also exhibit good wavelength

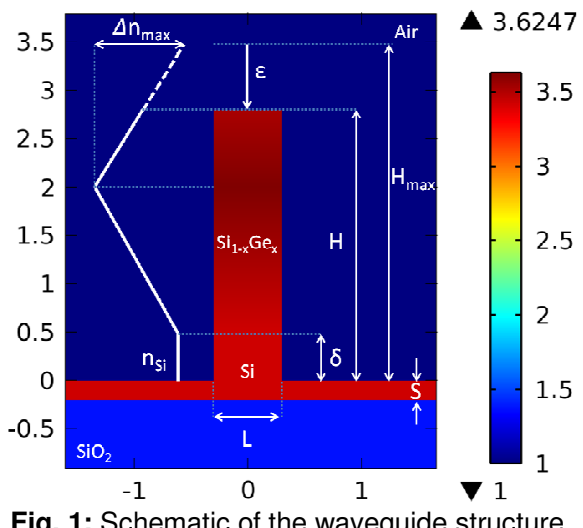

conversion performance for all-optical processing applications at telecommunication wavelengths. In this paper, we discuss the design aspects and fabrication of this type of devices and demonstrate the nonlinear conversion of a mid-IR signal to the near-IR.

\section{Design aspects and modeling}

The nonlinear wavelength conversion from the targeted mid-IR region $(4 \mu \mathrm{m}-5 \mu \mathrm{m})$ to the near$I R$ region is based on the four-wave mixing process pumped by a tunable and continuous wave source covering the $2-2.5 \mu \mathrm{m}$ wavelength region4. Therefore, the proposed waveguide must be able of carrying the mid-IR signal, the pump wave at the $2-2.5 \mu \mathrm{m}$ region as well as the idler near-IR product at the same time while still maintain: a) Good confinement with small effective mode area for intense nonlinearities $b$ ) 
low loss throughout the wavelength span and $c$ ) sufficient coupling with the dominant mid-IR sources, namely the quantum cascade la-sers (QCLs).

On top of the low index substrate a continuous Si slab layer of thickness $S=210 \mathrm{~nm}$ was introduced [see Fig.1] under the nonlinear waveguide. The waveguide itself was constituted of a buffer with pure $S i$ section of thickness $\delta$ surrounded by a SiGe section. This section exhibited a specific index profile, with a standard step index in the horizontal direction and a graded index profile in the vertical direction. The step-index fiber-like behavior of the waveguide allows the centralization of the optical field in the high $\mathrm{Ge}$ region where the Kerr coefficient $n_{2}$ is higher, thus enhancing its nonlinear properties. The graded profile in the vertical direction $(\mathrm{y})$ followed a symmetric double linear gradient, peaking in the middle of the distance $\left(H_{\max }-\delta\right)$. In the schematic, $L$ denotes the waveguide width and $H$ the total waveguide height after etching of the waveguide. $H$ corresponds to the initial deposition height $\left(H_{\max }\right)$ reduced by the quantity $\varepsilon$ after etching. The index would then normally vary from $n_{S i}$ at $\mathrm{y}=\delta$ to $n_{S i}+\Delta n_{\max }$ at $\mathrm{y}=\left(\delta+H_{\text {max }}\right) / 2$ and then back down to $n_{S i}$ at $\mathrm{y}=$ $H_{\max }$. The maximum allowed Ge concentration at $y=\left(\delta+H_{\max }\right) / 2$ was $42 \%$. Throughout the simulations $H_{\max }$ was kept constant at $3 \mu \mathrm{m}$. However, $H$ (translating to a combination of $\varepsilon$ and $\delta$, since $H=\delta+H_{\max }-\varepsilon$ ), $L$ and $S$ could be varied in order to properly engineer the waveguide's dispersion characteristics. The existence of a higher index core adjustable by geometrical characteristics proved to be a valuable tool in positioning the mode profile in the vertical direction. Calculations were performed using COMSOL's Finite Elements solver. The evolution of the effective index of the fundamental mode with wavelength was calculated by taking into account the material dispersion. Finally, the contribution of SiGe in each mode profile was estimated by calculating the integral:

$$
n=\frac{\iint n(\mathrm{x}, \mathrm{y})|E|^{2} d x d y}{\iint|E|^{2} d x d y}
$$

where $n(x, y)$ is the material wavelength dependent index over the $\mathrm{Si}$, SiGe plane.

The main factor affecting the waveguide dispersion is the ratio $H / L$. For a given $H$ value, an increase in $L$ results in a blue shift of the ZDW (see fig. 2). However by further increasing the waveguide width, the effective mode area becomes too large to support strong non-linear phenomena. An interesting concept ascends when varying the offset, $\delta$. As $\delta$ increases the waveguide tends to resemble a bulk $\mathrm{Si}$ wave-

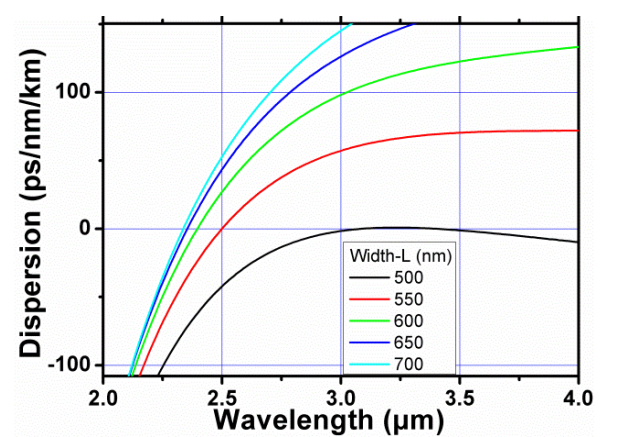

Fig. 2: Dispersion properties of the waveguide for 5 waveguide width values and height $H=2.05 \mu \mathrm{m}(\delta=1 \mu \mathrm{m})$

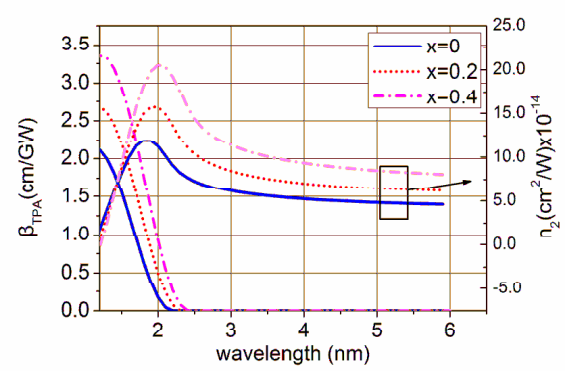

Fig. 3: Non-linear properties of $\mathrm{Si}_{1-x} \mathrm{Ge}_{\mathrm{x}}$ alloys

guide. Although higher overlap between the modes at different wavelengths occurs, the $\mathrm{Ge}$ contribution is reduced which results in weaker $\mathrm{Ge}$ induced non-linearity. As $\delta$ goes to zero the conditions are reversed.

The numerical model regarding the propagation of the pump, signal and idler obeys the nonlinear coupler propagation equations [6]. The critical parameters for the model are the Kerr coefficient $\mathrm{n}_{2}$, two photon absorption $\beta_{\text {TPA }}$, effective mode area, modal refractive index and mode overlap factors are known for all possible combinations [6]. The parameters $n_{2}$ and $\beta_{\text {TPA }}$ characterize the material. According to ref. 4, the Garcia's model fitted to available experimental measurements can be utilized to predict the $n_{2}, \beta_{\text {TPA }}$ evolution of $\mathrm{Si}_{1-x} \mathrm{Ge}_{\mathrm{x}}$ alloys for all the wavelengths of interest. Effective mode area, modal refractive index and mode overlaps can be calculated with COMSOL. Fig. 3 shows the calculated $n_{2}, \beta_{\text {TPA. }}$. As $x$ increases, the $n_{2}$ parameter, which is crucial for efficient parametric interaction, also increases and its maximum value shifts towards longer wavelengths. An $\mathrm{x}$ value between 0.0 to 0.4 reassures that pump wavelengths within 2 $2.4 \mu \mathrm{m}$ experience enhanced nonlinearity and therefore generate idler photons with higher efficiency. The designed waveguide exhibits the ZDW around this region, hence phase matching can be almost combined with maximum nonlinearity. 

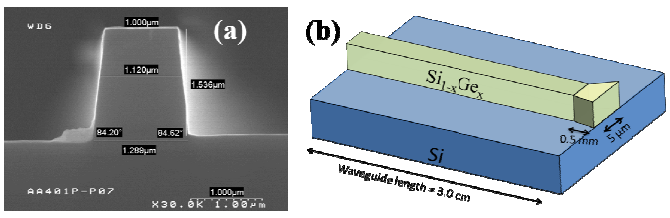

Fig. 4: a) Scanning Electron Microscopy (SEM) image of the SiGe waveguide, (b) Sketch of the SiGe waveguide

\section{Fabrication and Experiments}

The SiGe waveguide structure was grown by reduced pressure chemical vapor deposition on a SOI (silicon on insulator) wafer with $2 \mu \mathrm{m}$ buried oxide layer (Box). A buffer Si layer was first grown to adjust the SOI layer thickness to the desired value $(S+\delta)$. The linear gradient in germanium concentration on the waveguide profile was then achieved by varying the germane mass-flow during growth of the SiGe layer while keeping the dichlorosilane mass-flow constant. As a result, the concentration of $\mathrm{Ge}$ in the SiGe layer was linearly increased from $0 \%$ to $42 \%$ and then decreased back to pure $\mathrm{Si}$ symmetrically, as shown in Fig. 1(a). The top of the SiGe graded index layer was lowered by etching while monitoring in situ the Ge content in the etched by-products with mass spectroscopy. The process was stopped when the SiGe concentration was such that yielded the appropriate $\mathrm{H}$ value of $1.536 \mu \mathrm{m}$. Then the waveguide was realized by photolithography and etching. Etching ensured that the thickness of $\mathrm{Si}$ on the box layer that constituted the slab was $210 \mathrm{~nm}$. Figure 4(a) shows a scanning electron microscopy (SEM) image of the fabricated waveguide cross-section.

Fig. 5 summarizes our measurements and compares them with numerical simulations which considered an input launched power of 75 $\mathrm{mW}$ for the pump and $10 \mathrm{~mW}$ for the signal, and waveguide losses of $2 \mathrm{~dB} / \mathrm{cm}$, in line with the experimental values. The comparison is in qualitative agreement. The difference between the experimental and numerical data can be attributed to several factors, such as the exact dispersion characteristics of the waveguide. As an example, we show in Fig. 5 the difference that a shift of just $+6 \mathrm{~nm}$ (green dashed line) or $-6 \mathrm{~nm}$ (red dashed line) in the ZDW would make to the wavelength conversion performance. It was found that the nonlinear losses could be neglected for the pump powers considered in our experiments $(<100 \mathrm{~mW})$. The experimental characterization of the waveguide revealed one of the broadest wavelength conversion performances obtained for a signal at $2650 \mathrm{~nm}$ to an idler at $1771 \mathrm{~nm}$.

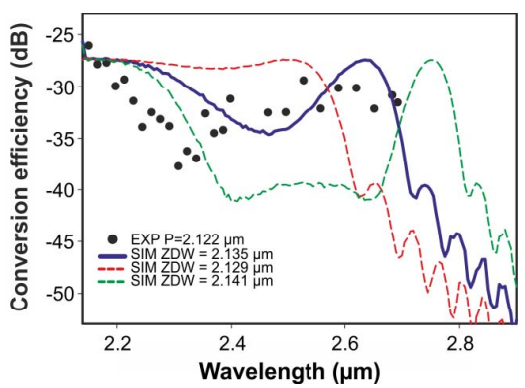

Fig. 4: a) Conversion efficiency as a function of the signal wavelength. Comparison between experiments (black dots) and numerical simulation for the dispersion shown in Fig. 2(a) (blue solid line) and for the zero dispersion shifted of $+6 \mathrm{~nm}$ (green dashed line) and - 6 $\mathrm{nm}$ (red dashed line).

\section{Conclusions}

We have reported the design, fabrication and characterization of SiGe waveguides tailored for the conversion of mid-infrared signals to the near-infrared based on four-wave mixing. The waveguide exhibited a linearly graded concentration in germanium, which allowed both for an enhanced nonlinear performance (as compared to pure Si waveguides) and a tailored ZDW. The broadest conversion we observed was from 2.65 $\mu \mathrm{m}$ to $1.77 \mu \mathrm{m}$ using a pump at $2.12 \mu \mathrm{m}$. The experiment demonstrates that dispersionengineered SiGe waveguides could lead to the realization of mid-IR spectrometers and highsensitivity sensors. The same technique can be applied for down-conversion as well, however limited by the onset of two-photon absorption at the signal wavelengths.

\section{Acknowledgements}

This work was supported by the European Communities Seventh Frame-work Program FP7/2007-2013 under Grant 288304 (STREP CLARITY).

\section{References}

[1] Y. Yao, et al, "Mid-infrared quantum cascade lasers," Nat. Photon. 6, 432-439 (2012).

[2] K. D. Buchter, et al, "Nonlinear optical down- and upconversion in PPLN waveguides for mid-infrared spectroscopy," in CLEO Europe (2009), CD_P8.

[3] B. Kuyken, et al, "Frequency conversion of mid-infrared optical signals into the telecom band using nonlinear silicon nanophotonic wires," OFC 2011, OThU4.

[4] N. K. Hon, e al, "The third-order nonlinear optical coefficients of $\mathrm{Si}, \mathrm{Ge}$, and Si1-xGex in the midwave and longwave infrared," J. Appl. Phys. 110, 011301 (2011).

[5] K. Hammani, et al, "Optical properties of silicon germanium waveguides at telecommunication wavelengths," Opt. Express 21, 16690-16701 (2013).

[6] Q. Lin, T. et al, "A proposal for highly tunable optical parametric oscillation in siliconmicro-resonators," Opt. Express 16, 10596-10610 (2008). 